\title{
The Overlap in the Genetic Pathogenesis of Ulcerative Colitis and Irritable Bowel Syndrome
}

\author{
Takeshi Kamiya
}

Published online: 14 November 2013

(c) Springer Science+Business Media New York 2013

Ulcerative colitis (UC) is a chronic, relapsing inflammatory disorder of the gastrointestinal tract. Although the etiology of UC remains unclear, genetic and environmental factors may contribute to its pathogenesis. Several genetic studies in Western and Eastern countries have reported associations between a number of polymorphisms and UC risk.

Irritable bowel syndrome (IBS) is one of the most common chronic functional bowel disorders in which abnormal discomfort or pain is associated with defecation or a change in bowel habits, in the absence of other disease that can explain such symptoms. Although gene-environment studies on IBS are lacking, disturbed gastrointestinal motility, dysbiosis of the colonic microbiome, sensory hypersensitivity, and psychosomatic factors have been attributed etiological significance.

Consensus exists that a shift in the host-gut microbial relationship leading to low-grade mucosal inflammation is contributory. IBS subjects have an increased number of inflammatory cells, including mast cells and lymphocytes, in mucosal biopsy samples obtained from the colon, rectum, and terminal ileum. Half of the patients with IBS have microscopic intestinal and colonic inflammation consistent with microscopic colitis. Moreover, acute infectious gastroenteritis is now recognized as an etiological factor for symptom development in a subset of patients with IBS, a condition termed post-infectious IBS (PI-IBS).

The original article, 10.1007/s10620-013-2677-y, published in the August 2013 issue of Digestive Diseases and Sciences on pp. 2314-2323.

T. Kamiya $(\bowtie)$

Department of Gastroenterology and Metabolism, Nagoya City

University Graduate School of Medical Science, Kawasumi 1,

Mizuho-cho, Mizuho-ku, Nagoya 467-8601, Japan

e-mail:kamitake@med.nagoya-cu.ac.jp
The main regulators of motor and sensory function of the gastrointestinal tract and a sentry cell guarding against invading microbes, mucosal mast cells are the most extensively studied immune cells in relation to IBS. The number of mast cells was significantly greater in the terminal ileum, cecum, colon, and rectum of the patients with IBS than in that of the healthy control groups [1, 2]. The concentration of mast cell mediators such as histamine, protease, and tryptase in supernatants from mucosal biopsies of patients with IBS was higher than in those from control groups. The release of these mediators may contribute to visceral hypersensitivity by activating the enteric nervous and sensory pain pathways.

The neurotransmitter 5-hydroxytryptamine $(5-\mathrm{HT})$ is present in enterochromaffin (EC) cells of the gastrointestinal mucosal epithelium or the serotonergic neurons of the enteric nervous system. 5-HT released from EC cells is a pro-inflammatory mediator that activates lymphocytes and enhances the secretion of several inflammatory cytokines. S100 proteins comprise a family of more than 20 proteins, which are endogenous activators of innate immune responses. S100A10, a very small protein of the S100 family, binds and increases surface expression of serotonin receptor subtype 5-HT1B and interacts with 5-HT4 receptors. Interleukin-10 (IL-10) is an anti-inflammatory cytokine that inhibits cytokine synthesis. Decreased IL-10 concentrations could predispose to increased mucosal cytokine production during infections or other mucosal insults. In human, a subset of inflammatory bowel diseases might be linked with IL-10 polymorphisms or to serum IL10 concentrations.

In an article previously published in this journal, Shiotani et al. [3] compared the rectal and sigmoid colonic mucosal expression of calgranulin proteins and functional polymorphisms of the 5-HT transporter and IL-10 genes in 
patients with diarrhea-predominant IBS (IBS-D) in comparison with UC or healthy controls. The authors reported that colonic mucosal expression of calgranulin A (S100A8 or myeloid-related protein 8; MRP8) and calgranulin B (S100A9; MRP14) was significantly higher in the UC than in that of IBS or healthy control groups and correlated with the UC disease activity index, that the expression of S100A10, proteins that co-localized with 5-HT1B receptors, was significantly higher in the rectal epithelium of patients with IBS than in the control group, and that this expression correlated with self-rating depression scale (SDS) scores; also, the frequency of the IL-10-819 CC genotype was significantly higher in patients with IBS-D and UC than in that of the control groups.

Therefore, it was concluded that rectal S100A10 overexpression may contribute towards IBS progression due to its involvement in the modulation of 5-HT1B receptors, and that IL10-819 CC is a candidate genotype for IBS and UC in Japanese populations.

Despite the novelty of these observations, they were not particularly surprising since patients with UC and IBS often share the symptoms of diarrhea and abdominal pain, and also because a subset of UC subjects without biochemical signs of active inflammation have ongoing intestinal symptoms that resemble those of IBS. Several studies report that IBS-like symptoms occur in one-third of patients with UC in remission, who display IBS-like gastrointestinal symptoms, despite no evidence of active inflammation. These UC subjects reportedly have several psychological symptoms and poorer quality of life compared with those in remission without gastrointestinal IBSlike symptoms. These findings suggest an association between UC and IBS pathogenesis, including the presence of occult inflammation in IBS subjects.

5-HT, highly concentrated in the gut, is thought to be an important contributor towards the pathogenesis of UC and IBS. Changes in intestinal EC cell numbers and mucosal 5-HT concentrations have been observed in experimental colitis and in patients with inflammatory bowel disease (IBD). Moreover, 5-HT is an important regulator of gastrointestinal motility, secretion, and sensation through specific receptors. In response to increased intestinal intraluminal pressure or chemical stimuli, EC cells release 5-HT, which stimulates intrinsic primary afferent neurons which initiate peristaltic and secretory reflexes. 5-HT is also involved in the transmission of sensory information from the gut lumen to the central nervous system. For all of these 5-HT properties, and since the number of EC cells and mucosal 5-HT concentrations are usually altered in IBS subjects, 5-HT may be a key component of IBS pathogenesis.

5-HT requires the serotonin reuptake transporter (SERT) for transport across the cell membrane prior to degradation. Therefore, a reduction in SERT function impairs intracellular uptake and degradation, increasing availability of 5-HT within the mucosa. Coates et al. [4] reported that mucosal 5-HT, tryptophan hydroxylase 1 messenger RNA (mRNA), SERT mRNA, and SERT immunoreactivity were all significantly reduced in rectal biopsy specimens of patients with UC, constipation-predominant IBS (IBS-C), and IBS-D compared with that of control groups. These data demonstrated that patients with UC and IBS shared similar molecular changes in serotonergic signaling mechanisms, and shared defects in 5-HT signaling which could plausibly alter motility, secretion, and sensation in the intestinal tract.

IL-10 is a key modulator for the maintenance of inflammation, attenuating immune system activation. Several observations point to IL-10 involvement in UC pathogenesis; IL-10 knockout mice develop colitis if they are kept in a germ-free environment [5]; IL-10 administration ameliorates colitis-related inflammation in animal models; and finally patients with UC are more likely to have genotypes associated with low IL-10 production [6].

Increased plasma concentrations of IL- 6 and IL- 8 and an abnormal IL-10/IL-12 were detected in IBS subjects. Increased interferon- $\gamma$ concentrations and decreased IL-10 concentrations were present in the intestinal mucosa of the patients with PI-IBS, suggesting that the pattern of response to gut microbes may affect the balance of $\mathrm{T}$ helper (Th) 1 and 2 cells. Yet, only a few studies have addressed the association of IL-10 and IL-10 gene polymorphisms in IBS. Chang et al. [7] reported lower colonic mucosal mRNA expression of IL-10 in IBS females compared with healthy females. Moreover, several IL-10 genotypes were less prevalent in patients with IBS compared with that of control groups [8]. These results suggested that some patients with IBS were genetically predisposed to produce lower amounts of IL-10 and can potentially develop mild inflammatory manifestations of IBS.

Haub et al. [9] demonstrated that SERT expression was significantly increased in inflamed colons of IL- $10^{-/-}$ (deficient) mice and that colitis associated with IL-10 ${ }^{-1-}$ mice was enhanced when IL-10 deficiency was combined with SERT deficiency, suggesting a relationship between 5-HT and IL-10. Nevertheless, further studies are required to investigate the role of biomarkers, such as S100A and IL-10-819CC, on 5-HT receptor and IL-10 polymorphisms for the indication and diagnosis of IBS and UC.

\section{References}

1. Ohman L, Simren M. Pathogenesis of IBS: role of inflammation, immunity and neuroimmune interactions. Nat Rev Gastroenterol Hepatol. 2010;7:163-170.

2. Barbara G, Stanghellini V, Cremon C, et al. Aminisalicylates and other anti-inflammatory compounds for irritable bowel syndrome. Dig Dis. 2009;27:115-121. 
3. Shiotani A, Kusunoki H, Kimura Y, et al. S100A expression and interleukin-10 polymorphisms are associated with ulcerative colitis and diarrhea predominant irritable bowel syndrome. Dig Dis Sci. 2013;58:2314-2323.

4. Coates MD, Mahonet CR, Linden DR, et al. Molecular defects in mucosal serotonin content and decreased serotonin reuptake transporter in ulcerative colitis and irritable bowel syndrome. Gastroenterology. 2004;126:1657-1664.

5. Kuhn R, Lohler J, Rennick D, et al. Interleukin-10 deficient mice develop chronic enterocolitis. Cell. 1993;75:263-274.

6. Togore A, Gonsaikarole WM, Pravica V, et al. Interleukin-10 (IL10) genotypes in inflammatory bowel disease. Tissue Antigens. 1999;54:386-390.
7. Chang L, Adeymo M, Karagiannides I, et al. Serum and colonic mucosal immune markers in irritable bowel syndrome. Am J Gastroenterol. 2012;107:262-272.

8. Gonsalkorale WM, Perrey C, Pravica V, et al. Interleukin 10 genotypes in irritable bowel syndrome: evidence for an inflammatory component? Gut. 2003;52:91-93.

9. Haub S, Ritze Y, Bergheim I, et al. Enhancement of intestinal inflammation in mice lacking interleukin 10 by deletion of the serotonin reuptake transporter. Neurogastroenterol Motil. 2010;22: 826-e229. 